\title{
Dynamical Adaptation of Parental Care
}

\author{
Yasuhiro Takeuchi*, Wendi Wang ${ }^{\dagger, a}$, Shinji Nakaoka ${ }^{\ddagger}, b$, Shingo Iwami ${ }^{*, c}$ \\ *: Graduate School of Science and Technology, Shizuoka University, \\ Hamamatsu 432-8561, Japan.
}

$\dagger$ : Key Laboratory of Eco-environments in Three Gorges Reservoir Region, School of Mathematics and Statistics, Southwest University,

Chongqing, 400715, P. R. China.

$\ddagger$ : Graduate School of Mathematical Sciences, The University of Tokyo,

3-8-1 Komaba, Meguro, Tokyo, 153-8914 Japan.

$a$ : The research was supported by Heiwa-Nakajima Fund and National Science Fund of China (No. 10571143).

b: The research was partly supported by the Sasakawa Scientific Research Grant from The Japan Science Society.

$c$ : The research was supported by Research Fellowships of the Japan Society for the Promotion of Science for Young Scientists. 


\begin{abstract}
Two models are proposed to simulate population growth of species with mature stage and immature stage in which there are parental cares for immatures. It is assumed that the protection of matures to their immatures reduces mortality of immatures at the cost of reduction of reproduction. Dynamical adaptation of parental cares is incorporated into the models, one of which is described with the proportional transition rate from immatures to matures (ODE model) and the other one is described with a transition rate from immatures to matures according to a fixed age (DDE model). For the ODE model, it is shown that the adaptation of parental care enlarges the possibility of species survival in the sense that population is permanent under the influences of the adaptation, but becomes extinct in the absence of adaptation. It is proved that the outcome of the adaptation makes the population in an optimal state. It is also observed that there are parental care switches, from non-care strategy to care strategy, as the natural death rate of immature individuals increases. The analysis of the DDE model indicates that the adaptation also enlarges the opportunity of population persistence, but the stage delay has the tendency to hinder the movement of population evolution to the optimal state. It is found that the loss rate of immatures in the absence of parental cares can induce different patterns to disturb the adaptation of population to optimal state. However, it is shown that the adaptation of parental care approaches to the optimal state when parental care is required for the survival of the population, for example, when the loss rate of immatures or competition among matures increases or the fecundity decreases.
\end{abstract}

Key Words: Parental care; Adaptation; Stage; Stability; Optimal. 


\section{Introduction}

Parental care is a fundamental biological activity because matures of many organisms provide their immature offsprings with food and protection. Biological studies of this topics are related to parental care patterns (uniparental care by either sex, sex-biased parental care, or biparental care) and parental care investments (Lessells, 1998; Seno and Endo, 2007; Yaniv, 2005). It is recognized that parental care increases offspring survival, but reduces parental fecundity and mating opportunities (Reynolds et al., 2002). Indeed, if a mature spends more time in searching food to have more benefits for reproductions, its immatures face more risks to be lost due to poor living conditions, or attack from enemies. These have been observed and studied from biological point of view including experiments. Bruno et al. (2007) conducted experimental manipulations to identify and quantify the costs and benefits of parental cares of the harvestman. They found that in a removal experiment of parental cares that lasted 14 days, larvae without parental cares survived $75.6 \%$ less than those with parental cares. By monitoring females' reproductive success for 2 years, they found that parents experimentally prevented from guarding their larvae produced new offspring more frequently and had mean lifetime fecundity $18 \%$ higher than that of control guarding parents.

There have been a few of mathematical considerations to find strategies of parental investments, among which are game analyses done in Forbes and Mock (1998) and Houston et al. (2005). It seems that game analysis is better applicable when population is in a steady state and individuals know all information to choose the best strategy. To consider dynamical influences of parental care, Wang, Nakaoka, and Takeuchi (2008) and Wang, Takeuchi, Saito, and Nakaoka (2006) proposed mathematical models that incorporate trade-offs between the protection of immatures and the reproduction of matures. However, all the parameters are constants over time in the models of Wang et al. (2008) and Wang et al. (2006), which means that the adaptation of parental care is ignored. Notice that parental care time is a behavioral strategy that can be adjusted by matures to contribute better fitness. Motivated by recent works of DeAngelis et al. (2006); Abrams (2007); Abrams et al. (2007); Krivan (2007), we propose two models with the adaptation of parental care where the parental care time is continuously adjusted to represent the natural selection of matures to achieve better fitness through learning favorable strategy. Our models have two stages (immature and mature), between which there is a transition from immature to mature. 
The organization of this paper is as follows. In the next section, we present the model formulation of a population with parental care described with a proportional transition rate from immature individuals to mature individuals. Our analysis shows that the adaptation of parental care enlarges the possibility of population survival in the sense that population is permanent under the influences of the adaptation, but becomes extinct if there is no adaptation. We also prove that the adaptation makes the population in an optimal state. In section 3, we introduce another mathematical model with the adaptation of parental care with the transition from immature individuals to mature individuals according to a fixed age. It is shown that the adaptation also enlarges the opportunity of population persistence, but the stage delay has the tendency to hinder the movement of population evolution to the optimal state.

\section{Model with proportional transition}

\section{$2.1 \quad$ Model}

To consider dynamical behavior of parental care, we use a stage-structured model that splits a population into two categories: immature class and mature class. Continuous stage structured models were proposed or studied by Nisbet and Gurney (1983), Aiello and Freedman (1990); Aiello et al. (1992), Thieme (2003), de Roos et al. (2003), Gourley and Kuang (2004, 2005), Kostova et al. (1999), Wang and Chen (1997); Wang et al. (2001); Wang and Zhao (2005, 2006). Basically, modeling approaches are divided into two types according to the rate of transition: ODE approach and DDE approach. We start from the ODE method. Let $y_{1}$ be the density of immature individuals, $y_{2}$ be the density of mature individuals, $c$ be the fraction of parental care time in one unit time and $s$ be the fraction of searching time in one unit time. Then $c+s=1$. Care time $c$ is a behavioral strategy for matures. As a trait, it can adapt and evolve because of learning of individuals. We hope to find influences of adaptation of care time $c$ on population dynamics.

To derive the recruitment rate of population, we assume that the resource input is constant $r$, which is a reasonable approximation in the first step and simplifies the dynamics. Moreover this assumption can be reasonable if the resouce recovers very fast to become steady state. Then one mature admits the resource $r / y_{2}$. If $a_{0}$ is the searching efficiency of the resource for one mature, since the fraction of searching time becomes $1-c$ because of parental care, the searching efficiency is 
now reduced to $a_{0}(1-c)$. As a result, the uptake rate of resource for one mature is $x=a_{0}(1-c) r / y_{2}$. To incorporate the saturation effect converting the nutrient $x$ into offsprings, we select the simplest candidate of Beverton-Holt recruitment function $\frac{k x}{1+h x}$ to obtain per capita birth rate of one mature:

$$
k \frac{a_{0}(1-c) \frac{r}{y_{2}}}{1+a_{0}(1-c) h \frac{r}{y_{2}}}=\frac{a(1-c)}{b(1-c)+y_{2}}
$$

with $a=k a_{0} r$ and $b=a_{0} h r$. Now, without evolution of $c$, we can assume that population dynamics is described by

$$
\left\{\begin{aligned}
\frac{d y_{1}}{d t} & =\frac{a(1-c) y_{2}}{b(1-c)+y_{2}}-d_{1} y_{1}-\alpha(1-c) y_{1}-\nu y_{1} \\
\frac{d y_{2}}{d t} & =\nu y_{1}-d_{2} y_{2}
\end{aligned}\right.
$$

where $\alpha$ gives the loss rate of immatures in the absence of parental cares per unit time, $d_{1}$ and $d_{2}$ are the natural per capita death rates of immature individuals and mature individuals and $\nu$ is the transition rate from immature stage to mature stage. That is, we assume that the average maturation time is $1 / \nu$. This is a simple way to model stage structures when ages of immature individuals are not easily measured or maturation is size-dependent (Thieme, 2003). Another adavantage of this methodology is that the transition rate from immature stage to mature stage can be easily obtained by statistical method such as linear regression.

To include adaptation of parental care in (2.1), we must define the fitness of the population. Without a stage structure, it is just the per capita net growth rate of the species (Metz et al., 1992; Geritz et al., 2004). Now, because one mature is a representative of one 'family' (one mature together with its immature members) and an adult should consider the future of whole family, its care time and reproduction are connected to a family. Thus, it is reasonable to consider that costs and benefits should be calculated according to a family. Hence, we define the fitness by

$$
F=\frac{a(1-c)}{b(1-c)+y_{2}}-\left(d_{1}+\alpha(1-c)\right) \frac{y_{1}}{y_{2}}-d_{2}
$$

Here note that

$$
F=\left(\frac{d y_{1}}{d t}+\frac{d y_{2}}{d t}\right) / y_{2}
$$


Following the spirit of adaptation (Dieckmann and Law, 1996; Hofbauer and Sigmund, 1998; Abrams, 2000), we assume that adaptation equation takes the form:

$$
\frac{d c}{d t}=\beta(c) \frac{\partial F}{\partial c}=\beta(c)\left(-\frac{a y_{2}}{\left(b(1-c)+y_{2}\right)^{2}}+\alpha \frac{y_{1}}{y_{2}}\right),
$$

where $\beta(c)$ satisfies: $\beta(0)=\beta(1)=0, \beta(c)>0$ for $0<c<1$ to ensure $c$ lies between 0 and 1 . Thus, the full model is

$$
\left\{\begin{aligned}
\frac{d y_{1}}{d t} & =\frac{a(1-c) y_{2}}{b(1-c)+y_{2}}-d_{1} y_{1}-\alpha(1-c) y_{1}-\nu y_{1}, \\
\frac{d y_{2}}{d t} & =\nu y_{1}-d_{2} y_{2}, \\
\frac{d c}{d t} & =\beta(c)\left(-\frac{a y_{2}}{\left(b(1-c)+y_{2}\right)^{2}}+\alpha \frac{y_{1}}{y_{2}}\right) .
\end{aligned}\right.
$$

To be specific, we choose $\beta(c)=w c(1-c)$ with a positive constant $w$. Indeed, mathematical analysis and computer simulations below show that the change of adaptation coefficient $\beta(c)$ does not influence qualitative behavior of (2.2), though the adaptation speed is altered. $w$ is always fixed at 1 for numerical computations.

We transform (2.2) by $u=y_{1} / y_{2}$ to obtain

$$
\left\{\begin{aligned}
\frac{d u}{d t} & =\frac{a(1-c)}{b(1-c)+y_{2}}-\left(d_{1}+\alpha(1-c)+\nu-d_{2}\right) u-\nu u^{2}, \\
\frac{d y_{2}}{d t} & =y_{2}\left(\nu u-d_{2}\right), \\
\frac{d c}{d t} & =w c(1-c)\left(-\frac{a y_{2}}{\left(b(1-c)+y_{2}\right)^{2}}+\alpha u\right) .
\end{aligned}\right.
$$

Note that (2.3) or (2.2) is defined only for $y_{2}>0$ because we consider the fitness according to a family of predators.

\subsection{Optimal versus Dynamical Adaptation}

Appendix A shows that (2.1) has a globally stable positive equilibrium point $E_{00}=$ $\left(\bar{y}_{1}, \bar{y}_{2}\right)$ when (A-3) is satisfied. On the other hand, adaptation system (2.2) has an asymptotically stable positive steady state $E^{*}=\left(y_{1}^{*}, y_{2}^{*}, c^{*}\right)$ when $(\mathrm{A}-6)$ is satisfied (see Theorem A in Appendix B and Table 1). Let us consider the relationship between $E_{00}$ and $E^{*}$. Let $f(s)=\bar{y}_{1}+\bar{y}_{2}$ be the total number of the population described by $(2.1)$ at globally stable steady state $E_{00}=\left(\bar{y}_{1}, \bar{y}_{2}\right)$. With $s=1-c$ we have

$$
f(s)=\left(1+\frac{\nu}{d_{2}}\right) \frac{a \nu-\left(d_{1}+\alpha s+\nu\right) b d_{2}}{\nu\left(d_{1}+\alpha s+\nu\right)} s .
$$


Function $f(s)$ attains a maximal value for $s \in\left(0, \frac{a \nu-b d_{2}\left(d_{1}+\nu\right)}{b d_{2} \alpha}\right)$ at

$$
s_{m}:=\frac{1}{\alpha}\left\{\sqrt{\frac{a \nu\left(d_{1}+\nu\right)}{b d_{2}}}-\left(d_{1}+\nu\right)\right\}=s^{*},
$$

where $s^{*}=1-c^{*}($ see $(\mathrm{A}-12))$. Thus, we have from (A-13)

$$
\begin{aligned}
f\left(s_{m}\right) & =\left(1+\frac{\nu}{d_{2}}\right) \frac{a \nu-b d_{2} \sqrt{\frac{a \nu\left(d_{1}+\nu\right)}{b d_{2}}}}{\nu \sqrt{\frac{a \nu\left(d_{1}+\nu\right)}{b d_{2}}}} s^{*} \\
& =\left(1+\frac{d_{2}}{\nu}\right)\left(\frac{a \nu}{d_{2}} \sqrt{\frac{b d_{2}}{a \nu\left(d_{1}+\nu\right)}}-b\right) s^{*} \\
& =\left(1+\frac{d_{2}}{\nu}\right) y_{2}^{*}=y_{1}^{*}+y_{2}^{*} .
\end{aligned}
$$

Therefore, the equilibrium $E^{*}=\left(y_{1}^{*}, y_{2}^{*}, c^{*}\right)$ of $(2.2)$ presents the optimal care time $c^{*}$ such that population size attains maximal value $y_{1}^{*}+y_{2}^{*}$. This means that the outcome of adaptation makes the population in optimal state.

Now, we can present that the adaptation of population enhances its persistence. Indeed, the case that $c=1$ is uninteresting because there is no reproduction. For $0 \leq c<1$, if we set

$$
\begin{array}{ll}
A_{0}=\frac{b d_{2}\left(d_{1}+\nu\right)}{\nu}, & A_{c}=\frac{b d_{2}\left(d_{1}+\alpha(1-c)+\nu\right)}{\nu}, \\
A_{1}=\frac{b d_{2}\left(d_{1}+\alpha+\nu\right)}{\nu}, & A_{2}=\frac{b d_{2}\left(d_{1}+\alpha+\nu\right)^{2}}{\nu\left(d_{1}+\nu\right)},
\end{array}
$$

it is easy to see

$$
A_{0}<A_{c} \leq A_{1}<A_{2} .
$$

Then Table 1 follows easily from comparison of existence and stability of positive equilibrium $E_{00}$ of (2.1) and equilibria $E_{1}$ and $E^{*}$ of (2.3) (see Appendix A and B). In fact, from Table 1 we obtain Figure 1. In region $B\left(A_{c}<a<A_{2}\right)$ and region $C$ $\left(A_{0}<a<A_{c}\right)$, positive equilibrium $E^{*}$ of $(2.3)$ that incorporates the adaptation of parental care is asymptotically stable. Moreover, numerical simulations show that positive equilibrium $E^{*}$ is globally stable (see Figure 2). Hence, the population survives in this case. However, since positive equilibrium $E_{00}$ of (2.1) does not exist in region $C$, the population without adaptation of parental care becomes extinct (see Appendix A). Therefore, the adaptation of the parental care enlarges the possibility of species survival. 


\begin{tabular}{|c|c|c|c|c|c|}
\hline$a$ & $a<A_{0}$ & $A_{0}<a<A_{c}$ & $A_{c}<a<A_{1}$ & $A_{1}<a<A_{2}$ & $A_{2}<a$ \\
\hline$E_{00}$ & no existence & no existence & existence(+) & existence( +$)$ & existence $(+)$ \\
\hline$E_{1}$ & no existence & no existence & no existence & existence(-) & existence $(+)$ \\
\hline$E^{*}$ & no existence & existence $(+)$ & existence( +$)$ & existence( +$)$ & no existence \\
\hline
\end{tabular}

Table 1: Comparison of existence and stability of equilibria of model (2.1) and model (2.3) where the sign - means the equilibrium is unstable and the sign + indicates the equilibrium is asymptotically stable (see Appendix A and B).

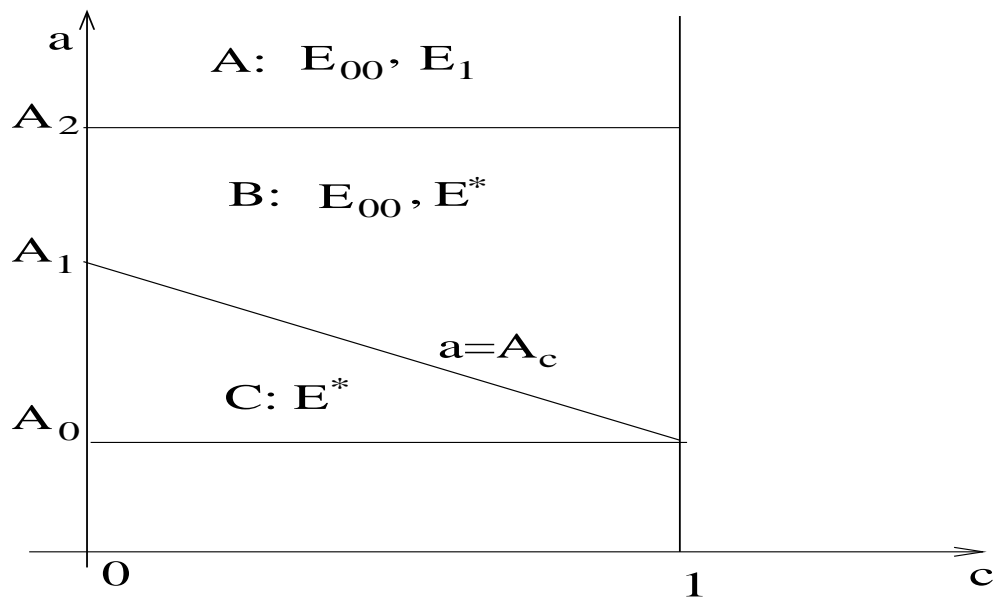

Figure 1: Distribution of equilibrium $E_{00}$ of (2.1) and equilibria $E_{1}$ and $E^{*}$ of (2.3) in the plane of $a$ (fecundity) and $c$ (parental care), where region $A$ gives "no relevance to adaptation", $B$ indicates "advantageous parental care" and $C$ presents "adaptation mediated survival".
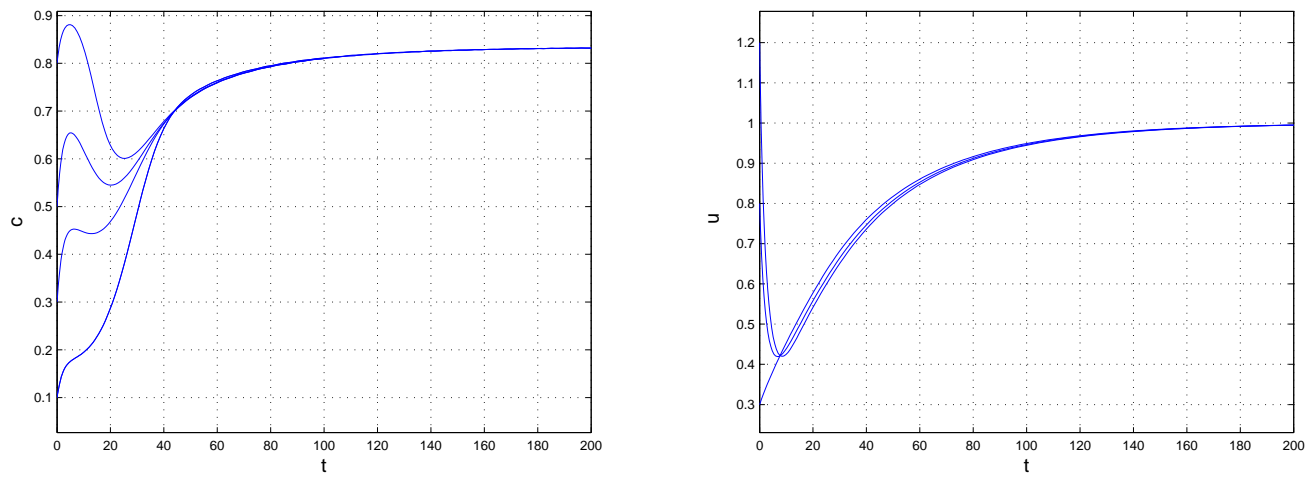

Figure 2: Graphs that show global stability of positive equilibrium $E^{*}$ of (2.3). 
We now reveal the mechanisms of parental care. In region $A\left(a>A_{2}\right), E_{00}$ and $E_{1}$ are stable. From the definition $a=k a_{0} r$, region $A$ implies that resource is rich ( $r$ is large), or searching efficiency of the species is high ( $a_{0}$ is large) or the conversion coefficient $k$ is large. In this case, having more reproductions is more important than reducing the loss of immatures, and hence parental care time $c(t) \rightarrow 0$ as $t \rightarrow \infty$, indicated in Figure 3 by arrows. On the other hand, in region $B$, the population adapts its strategy to the curve $1-c=s_{m}$ of optimal care time in order to achieve maximal population size. In region $C$ where the population becomes extinct without adaptation, the strategies of adaptation of care time are to move to the 'good' region $B$ first, and then approach to the curve of optimal care time, indicated by arrows in Figure 3.

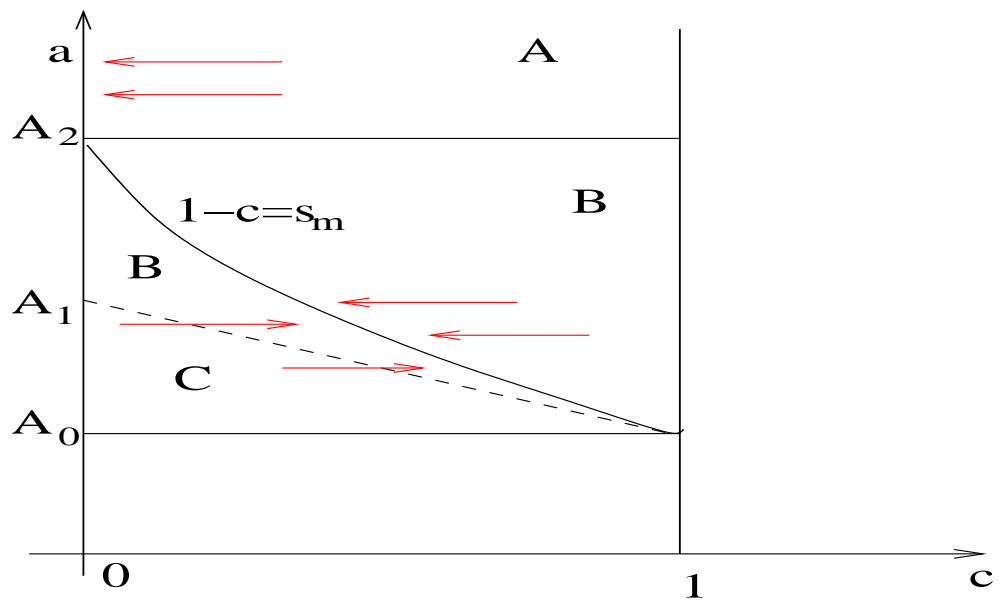

Figure 3: Strategies of parental care where arrows indicate the directions of reducing or increasing parental care times.

\subsection{Resource Competition and Parental Care}

We now point out that model (2.3) admits an interesting phenomenon of stability switches of equilibrium $E_{1}$ as the natural mortality rate $d_{1}$ of immatures varies. Indeed, let

$$
S=a-\frac{b d_{2}\left(d_{1}+\alpha+\nu\right)^{2}}{\nu\left(\nu+d_{1}\right)} .
$$

It follows from (A-14) that $E_{1}$ is stable if $S>0$, whereas $E_{1}$ is unstable if $S<0$. If we fix $b=2, d_{2}=0.1, a=18, \nu=0.1$ and $\alpha=2$, then we see that $E_{1}$ is unstable when $d_{1}<0.9$ or $d_{1}>3.9$, and is stable when $0.9<d_{1}<3.9$ by Table 1 (see the left panel of Figure 4). 
Notice that the change of stability of $E_{1}$ means the occurrence (at $d_{1}=3.9$ ) or disappearance (at $d_{1}=0.9$ ) of parental care. The stability switches imply that there are parental care switches as the natural death rate of immature individuals increases. The parental care is effective if the death rate of immatures is large (see the right panel of Figure 4 for $d_{1}>3.9$ ). Thus it is reasonable to see that $E_{1}$ is unstable on the region for larger $d_{1}$. It remains to know why the population needs to spend its time for parental care even though the death rate of immatures is small (see the right panel of Figure 4 for $d_{1}<0.9$ ). One possible explanation which could answer the question is the limitation of resource available to the population. If the death rate of immatures is small, a large number of them could be matured. It subsequently leads to the strong resource competition among matures, because of the Beverton-Holt functional response. Hence, the resource competition would decrease the effectiveness of searching food. We have a trade-off between the time for searching food and that for protecting immatures. The population is now advantageous to behave as spending more time to parental care.
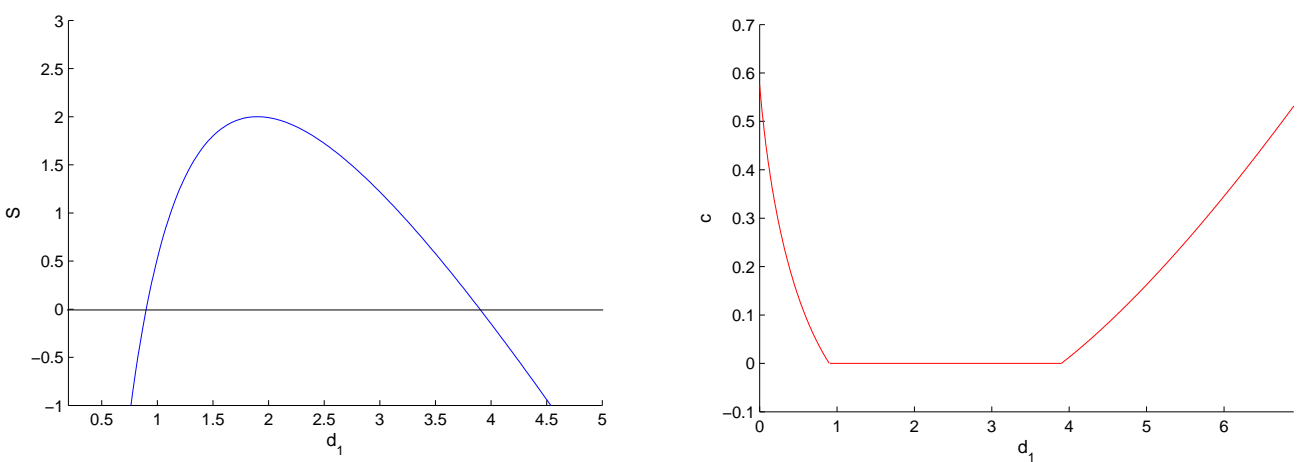

Figure 4: Left panel shows stability switches of $E_{1}$ and right panel indicates transitions of care times as $d_{1}$ increases.

\section{Model with transition by age}

\subsection{Model}

Another common way to model a stage structure is to use delayed differential equations (DDE) when the duration of immature stage can be easily measured. Following the modeling approach in the last section, without evolution of $c$, we 
assume that the population dynamics is described by

$$
\left\{\begin{array}{l}
\frac{d y_{1}}{d t}=\frac{a(1-c) y_{2}(t)}{b(1-c)+y_{2}(t)}-\left(d_{1}+\alpha(1-c)\right) y_{1}(t)-T, \\
\frac{d y_{2}}{d t}=T-d_{2} y_{2}(t),
\end{array}\right.
$$

where $T$ is the transition rate from immatures to matures, defined by

$$
\left.T=\frac{a(1-c) y_{2}(t-\tau)}{b(1-c)+y_{2}(t-\tau)} \exp \left\{-\left(d_{1}+\alpha(1-c)\right) \tau\right)\right\},
$$

in which $\tau$ gives the time for immatures to mature. Here $T$ represents the immatures born at time $t-\tau$ (with the mature birth $a(1-c) y_{2}(t-\tau) /\left\{b(1-c)+y_{2}(t-\tau)\right\}$ ) that survive to time $t$ (with the immature death rate $d_{1}+\alpha(1-c)$ ). This gives the immatures transition from the immature population entering the mature population at time $t$. The similar formulation can be found in many papers, for example, Aielo and Freedman (1990).

By similar arguments in the previous section, we introduce the adaptation equation

$$
\frac{d c}{d t}=w c(t)(1-c(t))\left(-\frac{a y_{2}(t)}{\left(b(1-c(t))+y_{2}(t)\right)^{2}}+\alpha \frac{y_{1}(t)}{y_{2}(t)}\right),
$$

and obtain the full model:

$$
\left\{\begin{aligned}
\frac{d y_{1}}{d t}= & \frac{a(1-c(t)) y_{2}(t)}{b(1-c(t))+y_{2}(t)}-\left(d_{1}+\alpha(1-c(t))\right) y_{1}(t) \\
& -\frac{a(1-c(t-\tau)) y_{2}(t-\tau)}{b(1-c(t-\tau))+y_{2}(t-\tau)} \exp \left\{-\int_{t-\tau}^{t}\left(d_{1}+\alpha(1-c(\theta))\right) d \theta\right\}, \\
\frac{d y_{2}}{d t}= & \frac{a(1-c(t-\tau)) y_{2}(t-\tau)}{b(1-c(t-\tau))+y_{2}(t-\tau)} \exp \left\{-\int_{t-\tau}^{t}\left(d_{1}+\alpha(1-c(\theta))\right) d \theta\right\}-d_{2} y_{2}(t), \\
\frac{d c}{d t}= & w c(t)(1-c(t))\left(-\frac{a y_{2}(t)}{\left(b(1-c(t))+y_{2}(t)\right)^{2}}+\alpha \frac{y_{1}(t)}{y_{2}(t)}\right) .
\end{aligned}\right.
$$

Note that $c$ is now time dependent and we need the transition term expressed with integral form in the above.

\subsection{Optimal versus Dynamical Adaptation}

Table 2 presents the distribution of the equilibria of $(3.2)$. Here $E_{0}=(0,0,0)$ is an extinct steady state, $E_{1}=\left(y_{10}, y_{20}, 0\right)$ is an equilibrium state without parental care and $E^{*}\left(y_{1}^{*}, y_{2}^{*}, c^{*}\right)$ is one with parental care for (3.2), respectively (see Appendix $\mathrm{C}$ and Theorem B). See further the definition of $\tau^{*}, \tau^{* *}, \tau_{0}$ in Appendix C. 


\begin{tabular}{|c|c|c|c|c|}
\hline$\tau$ & $\tau<\tau^{*}$ & $\tau^{*}<\tau<\tau_{0}$ & $\tau_{0}<\tau<\tau^{* *}$ & $\tau^{* *}<\tau$ \\
\hline$E_{0}$ & no existence & no existence & no existence & existence \\
\hline$E_{1}$ & existence $(+)$ & existence $(-)$ & no existence & no existence \\
\hline$E^{*}$ & no existence & existence & existence & no existence \\
\hline
\end{tabular}

Table 2: Equilibria of model (3.2) as the stage delay varies, where the sign - means the equilibrium is unstable and the sign + indicates the equilibrium is asymptotically stable (see Appendix C).

Numerical calculations show that $E_{0}$ is globally stable when $\tau^{* *}<\tau, E^{*}$ is globally stable whenever it exists, and $E_{1}$ is globally stable for $\tau<\tau^{*}$ (see Figures 5 and 6$)$. Thus, there is no need for parental care when the stage delay is small
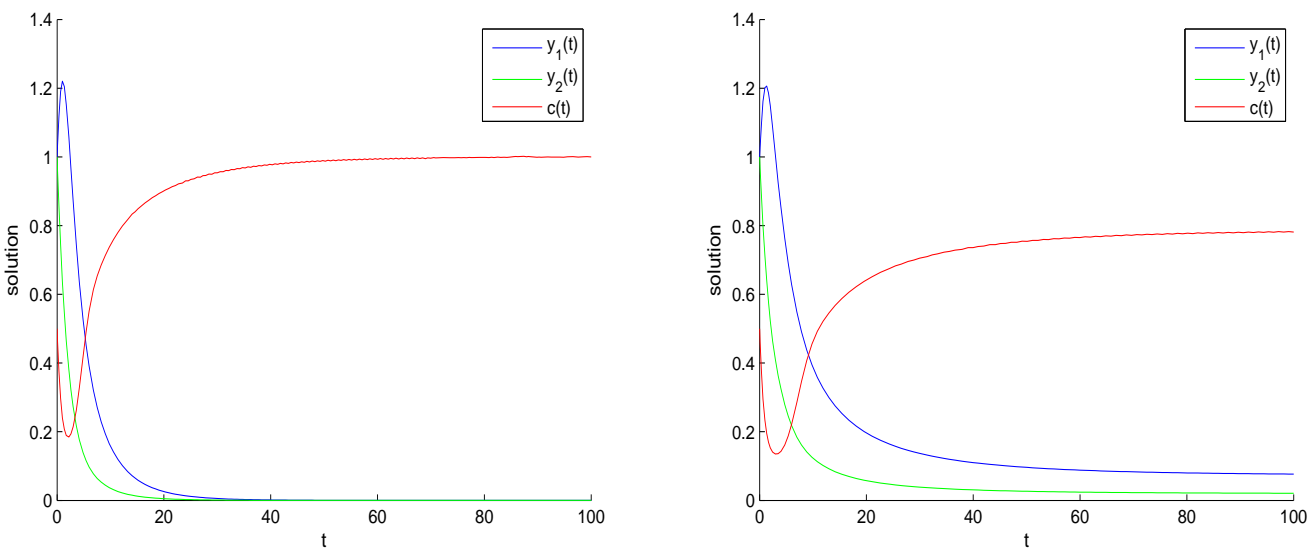

Figure 5: Graphs that show global stability of $E_{0}$ and $E^{*}$ of (3.2). The parameters for the graphs are fixed by $a=5, d_{1}=1, d_{2}=0.5, \alpha=0.5, b=1\left(\tau_{0}=1.54\right.$, $\tau^{*}=1.31$ and $\left.\tau^{* *}=2.30\right)$. The delay for the left panel is taken as $\tau=3.0>\tau^{* *}$ and the delay for the right panel is given by $\tau=2.0\left(\tau_{0}<\tau<\tau^{* *}\right)$.

(ref. the right panel of Fig. 6), and parental care is necessary when the stage delay is in a medium size (ref. the right panel of Fig. 5 and the left panel of Fig. 6).

To see whether the adaptation can enlarge the possibility of population survival, we consider the stability of model (3.1) where there is no population adaptation. Let

$$
\tau<\frac{1}{d_{1}+\alpha(1-c)} \ln \frac{a}{b d_{2}}:=\tau_{c} .
$$

(3.1) admits a globally stable positive equilibrium $E_{01}=\left(y_{01}, y_{02}\right)$ where

$$
\begin{aligned}
& y_{02}=\frac{a(1-c) e^{-\left(d_{1}+\alpha(1-c)\right) \tau}}{d_{2}}-b(1-c), \\
& y_{01}=\frac{a(1-c) y_{02}}{\left(b(1-c)+y_{02}\right)\left(d_{1}+\alpha(1-c)\right)}\left(1-e^{-\left(d_{1}+\alpha(1-c)\right) \tau}\right) .
\end{aligned}
$$



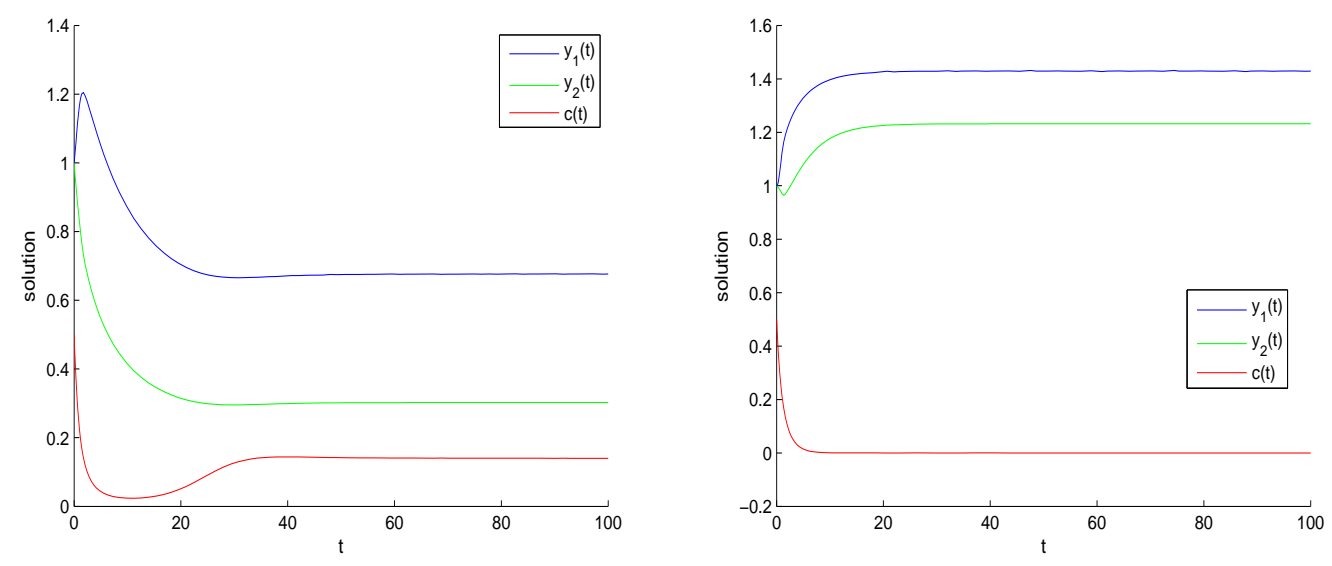

Figure 6: Graphs that show global stability of $E^{*}$ and $E_{1}$ of (3.2). The parameters are fixed as the same as Figure $5\left(\tau_{0}=1.54, \tau^{*}=1.31\right.$ and $\left.\tau^{* *}=2.30\right)$. The delay for the left panel is taken as $\tau=1.4\left(\tau^{*}<\tau<\tau_{0}\right)$ and the delay for the right panel is given by $\tau=1\left(\tau<\tau^{*}\right)$.

Furthermore, if $\tau>\tau_{c}$, positive solutions of (3.1) approach $(0,0)$ as $t \rightarrow \infty$. Note that $\tau_{c}<\tau^{* *}$ when $0 \leq c<1$, and that the population in (3.2) can survive when $\tau_{c} \leq \tau<\tau^{* *}$ (see Table 2). It follows that the adaptation increases the possibility of population persistence.

Motivated by the observations on ODE model (2.3), we hope to know whether the adaptation of population makes the population in an optimal state in the sense that $y_{1}^{*}+y_{2}^{*}$ gives the maximal value of $y_{01}(c)+y_{02}(c)$ when $c=c^{*}$. We assume that $y_{01}(c)+y_{02}(c)$ achieves a maximum value at $c=\bar{c}$. Note that $y_{1}^{*}$ and $y_{2}^{*}$ satisfy (A-23) and (A-24). If we fix $c=c^{*}=1-s^{*}$ in (3.4) with $s^{*}$ satisfying $f\left(s^{*}\right)=g\left(s^{*}\right)$ in the proof of Theorem B, we obtain $y_{1}^{*}=y_{01}\left(c^{*}\right)$ and $y_{2}^{*}=y_{02}\left(c^{*}\right)$. Hence, we have

$$
y_{1}^{*}+y_{2}^{*} \leq y_{01}(\bar{c})+y_{02}(\bar{c}),
$$

and the inequality holds unless $c^{*}=\bar{c}$. The following example shows that the population size at the positive equilibrium is less than the optimal population size.

Example 1 We take $\tau=1, \alpha=1, b=0.5, d_{1}=0.2, d_{2}=0.5, a=0.8$. By (3.4) we obtain

$$
\begin{aligned}
& y_{01}=\frac{0.5(1.6 \exp (-0.2-s) s-0.5 s)(1-\exp (-0.2-s))}{\exp (-0.2-s)(0.2+s)}, \\
& y_{02}=1.6 \exp (-0.2-s) s-0.5 s
\end{aligned}
$$

with $s=1-c$. Then $y_{01}+y_{02}$ achieves a maximal value 0.2572 at $s=0.4453$. 
However, we have $y_{1}^{*}=0.1065, y_{2}^{*}=0.1463, s^{*}=0.5093$. Since $y_{1}^{*}+y_{2}^{*}=0.2528<$ 0.2572 , the evolution of the population does not arrive at the ideal state.

\subsection{Effects of Stage Delay on Parental Care}

Example 1 shows that the stage delay has the tendency to reduce the opportunity for the population to move to the optimal state. To see influences of stage delay on the outcomes of adaptation, we first consider the effect of the stage delay $\tau$ on $y_{01}$ and $y_{02}$. By direct calculations, we obtain

$$
\begin{aligned}
& y_{02}^{\prime}(\tau)=-\frac{a s}{d_{2}}\left(d_{1}+\alpha s\right) e^{-\left(d_{1}+\alpha s\right) \tau}<0, \\
& y_{01}^{\prime}(\tau)=\left(a e^{-2\left(d_{1}+\alpha s\right) \tau}-b d_{2}\right) e^{\left(d_{1}+\alpha s\right) \tau} s
\end{aligned}
$$

and

$$
y_{01}^{\prime}(\tau)+y_{02}^{\prime}(\tau)=\left\{a\left(1-\frac{d_{1}+\alpha s}{d_{2}}\right) e^{-\left(d_{1}+\alpha s\right) \tau}-b d_{2} e^{\left(d_{1}+\alpha s\right) \tau}\right\} s .
$$

Hence, the density of matures at the equilibrium is a decreasing function of $\tau$, and the density of immature individuals at the equilibrium increases for $0<\tau<\tau_{c} / 2$, decreases for $\tau_{c} / 2<\tau<\tau_{c}$. Further, the density of whole population at the equilibrium increases for

$$
0<\tau<\frac{1}{2\left(d_{1}+\alpha s\right)} \ln \left\{\frac{a}{b d_{2}}\left(1-\frac{d_{1}+\alpha s}{d_{2}}\right)\right\},
$$

and decreases for

$$
\frac{1}{2\left(d_{1}+\alpha s\right)} \ln \left\{\frac{a}{b d_{2}}\left(1-\frac{d_{1}+\alpha s}{d_{2}}\right)\right\}<\tau<\tau_{c}
$$

(see Figure 7). From Figure 7, we see that parental care time $\bar{c}$, which gives the maximal total population size, increases as the stage delay increases.

Next, we consider the influences of stage delay on the difference between the optimal population size and the population size at the positive equilibrium with the adaptation. If we fix $a=3.0, b=0.5, d_{1}=0.1, d_{2}=0.6$ and vary $\alpha$, we obtain three different types of figures that show interesting phenomena about influences of stage delay on the adaptation (see Figure 8). As the loss rate $\alpha$ of immatures in the absence of parental cares becomes larger, the conflicts between reproduction and parental care become more severe. As a result, the curve for the difference, i.e., $y_{01}(\bar{c})+y_{02}(\bar{c})-\left(y_{1}^{*}+y_{2}^{*}\right)$, undergoes the transitions from a decreasing manner to the shape with one hump (increasing first, and then decreasing), and then to the shape with two humps. We examine how the difference can be influenced as 


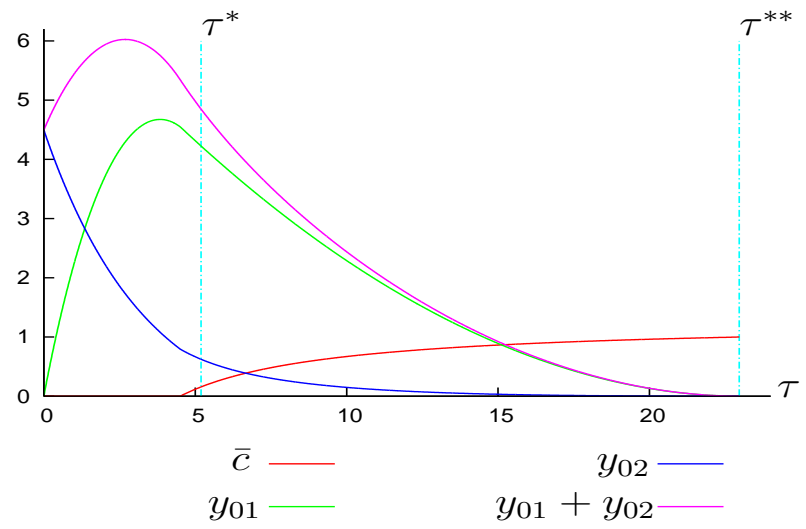

Figure 7: The graph of $y_{01}(\bar{c}), y_{02}(\bar{c}), y_{01}(\bar{c})+y_{02}(\bar{c})$ and $\bar{c}$ versus $\tau$ where $\alpha=$ $0.2, b=0.5, d_{1}=0.1, d_{2}=0.6, a=3.0$.
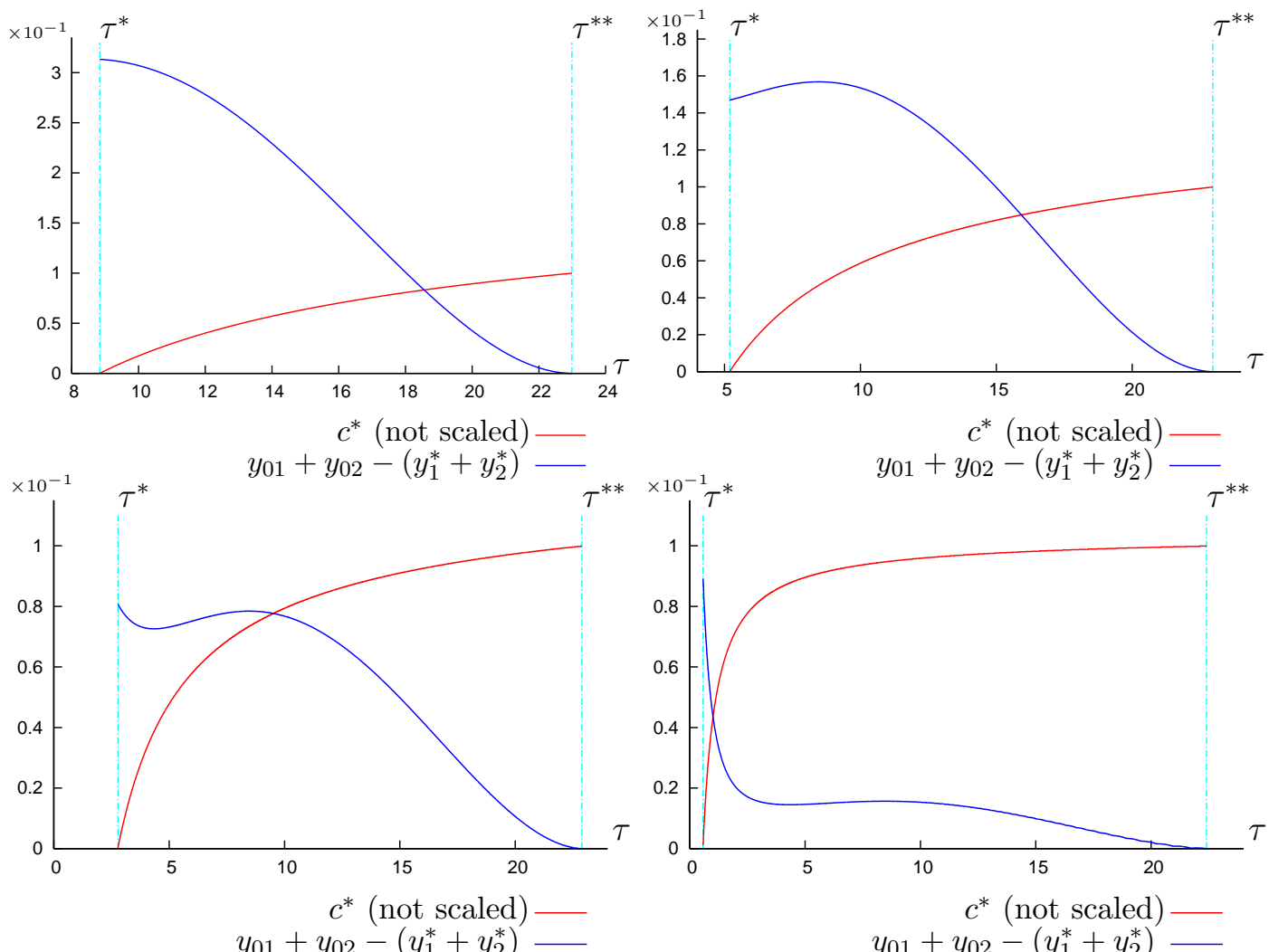

Figure 8: Graphs that show the adapted parental care time $c^{*}$ and differences of $y_{01}(\bar{c})+y_{02}(\bar{c})-\left(y_{1}^{*}+y_{2}^{*}\right)$ where $\alpha=0.1$ for the top left panel, $\alpha=0.2$ for the top right panel, $\alpha=0.4$ for the bottom left panel, and $\alpha=2.0$ for the bottom right panel. The other parameters are always fixed as $a=3.0, b=0.5, d_{1}=0.1$ and $d_{2}=0.6$. 

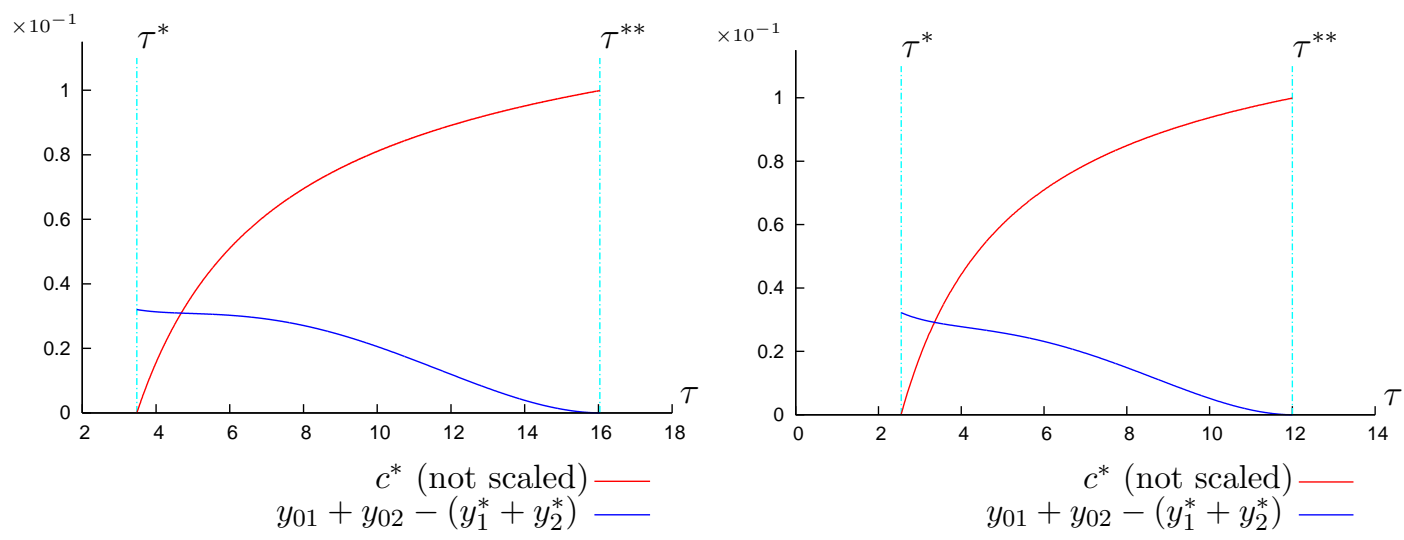

Figure 9: Graphs that show the adapted parental care time $c^{*}$ and differences of $y_{01}(\bar{c})+y_{02}(\bar{c})-\left(y_{1}^{*}+y_{2}^{*}\right)$ where $a=1.5$ and $b=0.5$ for the left panel whereas $a=3.0$ and $b=1.5$ for the right panel. The other parameters are fixed as same as one of the panel in Figure $8 ; \alpha=0.2, d_{1}=0.1$ and $d_{2}=0.6$.

the loss rate $\alpha$ of immatures varies. Among four panels in Figure 8, we observe that $\tau^{*}$ becomes smaller as $\alpha$ increases (c.f., (A-28)). The adapted parental care time $c^{*}$ rapidly saturates to 1 as $\alpha$ increases. Thus matures are forced to spend more time to care for immatures even though stage delay is small. Moreover, the difference $y_{01}(\bar{c})+y_{02}(\bar{c})-\left(y_{1}^{*}+y_{2}^{*}\right)$ becomes smaller as $\alpha$ increases, in the sense that the difference rapidly decreases to become small for almost stage delay.

Now we consider the influences of fecundity $a$ and competition among matures $b$ on the adaptation of parental care time. Compare the top right panel of Figure 8 and the left panel of Figure 9, where the latter panel has a smaller value of fecundity $a$. We observe that $\tau^{*}$ and the difference $y_{01}(\bar{c})+y_{02}(\bar{c})-\left(y_{1}^{*}+y_{2}^{*}\right)$ become smaller and $c^{*}$ rapidly saturates to 1 when fecundity is smaller. The same tendencies are also observed for larger competition among matures (compare the top right panel of Figure 8 and the right panel of Figure 9).

Note that adaptation is rapid if the right hand side of the adaptation equation in (3.2) is large. Above observations together with the adaptation equation in (3.2) suggest that the population undergoes adaptation toward increasing parental care, provided that either $\alpha$ is large, $a$ is small or $b$ is large. In other words, the more parental care is required, the smaller the difference becomes. Hence the population state approaches to the optimal state as parental care is required for the survival of the population. 


\section{Discussions}

In this paper, by dividing a population into immature stage and mature stage, we have proposed two models with stage structures to simulate the adaptation of dynamical parental care of matures to their offsprings. We have defined the population fitness according to families. Then we have given the rule of adaptation that matures adjust their care times according to the direction of gradient of fitness. For the proportional transition case (ODE model), we have shown that this is a perfect way of adaptation because the adaptation makes the population in an optimal state, and the adaptation of parental care enlarges the possibility of species survival in the sense that population is permanent under the influences of the adaptation, but becomes extinct in the absence of adaptation. We have also found the counterintuitive phenomenon that lower death rate of immature individuals induce parental care. For the model with transition by age (DDE model), we have verified that the adaptation also enlarges the opportunity of population persistence, but the stage delay has the tendency to hinder the movement of population evolution to the optimal state. Biological mechanism behind this is that the model with the stage structure induces trade-off between matures and immature members. The model with the proportional transition rate admits the nice property that mature individuals achieve optimal state together with their children. However, the model with the transition from immature individuals to mature individuals according to a fixed age has the nonlinear transition rate, which produces certain gap between the optimal occurrence of matures and the optimal occurrence of immatures. The numerical simulation suggests that the gap decreases as the loss of the immatures without parental care increases or the fecundity decreases or competition among matures increases. Under these circumstances, the adaptation approaches to the optimal state.

\section{APPENDIX}

\section{APPENDIX A}

\section{Stability of Equilibria of (2.1)}

We define $\frac{a(1-c) y_{2}}{b(1-c)+y_{2}}=0$ at $(0,0)$ since the reproduction rate is 0 when $y_{2}=0$ and $c=1$. Then (2.1) is well defined for $y_{1} \geq 0, y_{2} \geq 0$ and $0 \leq c \leq 1$. Note that 
(2.1) is a cooperative system when $0 \leq c<1$. Linearizing $(2.1)$ at $(0,0)$, we obtain

$$
\left\{\begin{array}{l}
\frac{d y_{1}}{d t}=-\left(d_{1}+\alpha(1-c)+\nu\right) y_{1}+\frac{a}{b} y_{2}, \\
\frac{d y_{2}}{d t}=\nu y_{1}-d_{2} y_{2} .
\end{array}\right.
$$

The coefficient matrix of the right-hand side of (A-1) has a negative dominant eigenvalue if

$$
d_{2} d_{1} b+d_{2} \alpha b(1-c)+d_{2} \nu b>a \nu,
$$

and has a positive dominant eigenvalue if

$$
d_{2} d_{1} b+d_{2} \alpha b(1-c)+d_{2} \nu b<a \nu .
$$

It follows from similar arguments (Takeuchi et al., 2006) that system (2.1) exhibits extinct of the population if (A-2) holds, and the population is persistent if (A-3) holds. Indeed, the theory of monotonic flows (Smith, 1995; Zhao, 2003) implies that system (2.1) has a positive equilibrium, denoted by $E_{00}$, that is globally stable.

\section{APPENDIX B}

\section{Stability of Equilibria of (2.3)}

First, we consider the case where $c=0$. Let $u_{0}$ be the unique positive solution of the following equation:

$$
\frac{a}{b}-\left(d_{1}+\alpha+\nu-d_{2}\right) u-\nu u^{2}=0 .
$$

Then $E_{0}=\left(u_{0}, 0,0\right)$ is an equilibrium point of $(2.3)$. When

$$
a \nu>d_{2} b\left(d_{1}+\alpha+\nu\right),
$$

another equilibrium $E_{1}=\left(\frac{d_{2}}{\nu}, y_{20}, 0\right)$ occurs where

$$
y_{20}=\frac{a \nu-d_{2} b\left(d_{1}+\alpha+\nu\right)}{d_{2}\left(d_{1}+\alpha+\nu\right)} .
$$

It is easy to see that $E_{0}$ is a saddle point. The stability of $E_{1}$ is not trivial. The Jacobian matrix at $E_{1}$ is

$$
\left[\begin{array}{ccc}
-d_{1}-\alpha-\nu-d_{2} & -\frac{a}{\left(b+y_{20}\right)^{2}} & -\frac{a}{b+y_{20}}+\frac{a b}{\left(b+y_{20}\right)^{2}}+\frac{\alpha d_{2}}{\nu} \\
\nu y_{20} & 0 & 0 \\
0 & 0 & w\left(-\frac{a y_{20}}{\left(b+y_{20}\right)^{2}}+\frac{\alpha d_{2}}{\nu}\right)
\end{array}\right]
$$


The characteristic equation is

$$
\left(\lambda-w\left(-\frac{a y_{20}}{\left(b+y_{20}\right)^{2}}+\frac{\alpha d_{2}}{\nu}\right)\right)\left(\left(d_{1}+\alpha+\nu+d_{2}+\lambda\right) \lambda+\frac{a \nu y_{20}}{\left(b+y_{20}\right)^{2}}\right)=0 .
$$

Hence, $E_{1}$ is asymptotically stable if

$$
\frac{a y_{20}}{\left(b+y_{20}\right)^{2}}>\frac{\alpha d_{2}}{\nu}
$$

while $E_{1}$ is unstable if

$$
\frac{a y_{20}}{\left(b+y_{20}\right)^{2}}<\frac{\alpha d_{2}}{\nu}
$$

Theorem A: (2.3) has a unique asymptotically stable positive equilibrium $E^{*}=$ $\left(u^{*}, y_{2}^{*}, c^{*}\right)$ if we have

$$
b d_{2}\left(d_{1}+\nu\right)<a \nu<\frac{b d_{2}\left(d_{1}+\nu+\alpha\right)^{2}}{d_{1}+\nu} .
$$

\section{Proof:}

If $E^{*}=\left(u^{*}, y_{2}^{*}, c^{*}\right)$ is a positive equilibrium of $(2.3)$, by the second equation of (2.3) we obtain $u^{*}=\frac{d_{2}}{\nu}$. Set $s^{*}=1-c^{*}$. By the third equation of (2.3), we have

$$
\frac{a y_{2}^{*}}{\left(b s^{*}+y_{2}^{*}\right)^{2}}=\alpha u^{*}
$$

Further, the first equation of (2.3) gives

$$
\begin{aligned}
\frac{a s^{*}}{b s^{*}+y_{2}^{*}} & =\left(d_{1}+\alpha s^{*}+\nu-d_{2}+\nu u^{*}\right) u^{*} \\
& =\left(d_{1}+\alpha s^{*}+\nu\right) u^{*} .
\end{aligned}
$$

61 Then (A-7) and (A-8) imply

$$
\frac{a}{\alpha u^{*}}\left(\frac{a s^{*}}{\left(d_{1}+\alpha s^{*}+\nu\right) u^{*}}-b s^{*}\right)=\frac{a^{2}\left(s^{*}\right)^{2}}{\left(d_{1}+\alpha s^{*}+\nu\right)^{2}\left(u^{*}\right)^{2}},
$$

which leads to

$$
a\left(d_{1}+\alpha s^{*}+\nu\right)-b\left(d_{1}+\alpha s^{*}+\nu\right)^{2} u^{*}=a \alpha s^{*} .
$$

363 A simplification of (A-10) yields

$$
b\left(d_{1}+\alpha s^{*}+\nu\right)^{2} u^{*}=a\left(d_{1}+\nu\right) .
$$


and

$$
y_{2}^{*}=\left(\sqrt{\frac{a b}{u^{*}\left(d_{1}+\nu\right)}}-b\right) s^{*} .
$$

$J 69:=\left[\begin{array}{c}-\left(d_{1}+\alpha\left(1-c^{*}\right)+\nu-d_{2}\right)-2 \nu u^{*} \\ \nu y_{2}^{*} \\ \alpha w c^{*}\left(1-c^{*}\right)\end{array}\right.$

where

The Jacobian matrix at $E^{*}$ is

$$
s^{*}=\frac{1}{\alpha}\left\{\sqrt{\frac{a\left(d_{1}+\nu\right)}{b u^{*}}}-\left(d_{1}+\nu\right)\right\},
$$

Note that (A-6) implies that $y_{2}^{*}>0$ and $0<s^{*}<1$. Therefore, the existence and uniqueness of the positive equilibrium is verified.

$$
\chi=-w c^{*}\left(1-c^{*}\right) \frac{2 a b y_{2}^{*}}{\left(b\left(1-c^{*}\right)+y_{2}^{*}\right)^{3}}<0 .
$$

Note that

$$
-\left(d_{1}+\alpha\left(1-c^{*}\right)+\nu-d_{2}\right)-2 \nu u^{*}=-\left(d_{1}+\alpha\left(1-c^{*}\right)+\nu\right)-\nu u^{*}<0 .
$$

It is easy to see that all characteristic roots of $J$ have negative real parts. The proof is complete.

We now give interesting comparisons between the dynamics of model (2.3) and the dynamics of model $(2.1)$. Let $E_{00}=\left(\bar{y}_{1}, \bar{y}_{2}\right)$ be the globally stable equilibrium of (2.1). Note that $\bar{y}_{2}=\frac{\nu}{d_{2}} \bar{y}_{1}$ and

$$
\bar{y}_{1}=(1-c) \frac{a \nu-\left(d_{1}+\alpha(1-c)+\nu\right) b d_{2}}{\nu\left(d_{1}+\alpha(1-c)+\nu\right)} .
$$

This equilibrium exists if and only if (A-3) holds.

Now we consider relations among stability conditions of various equilibria of (2.3). Note that the second inequality in (A-6)

$$
a \nu<\frac{b d_{2}\left(d_{1}+\nu+\alpha\right)^{2}}{d_{1}+\nu}
$$


is equivalent to (A-5). In fact, from the definition of $y_{20}$, we have

$$
y_{20}+b=\frac{a \nu}{d_{2}\left(d_{1}+\alpha+\nu\right)},
$$

which, together (A-14), implies

$$
\frac{a y_{20}}{\left(b+y_{20}\right)^{2}}=\frac{d_{2}\left(d_{1}+\alpha+\nu\right)\left(a \nu-b d_{2}\left(d_{1}+\alpha+\nu\right)\right)}{a \nu^{2}}<\frac{\alpha d_{2}}{\nu} .
$$

Thus, (A-14) leads to (A-5). The fact that (A-5) implies (A-14) can be easily obtained from above inequality. Hence, $E^{*}$ is asymptotically stable under conditions $(\mathrm{A}-5)$ and $b d_{2}\left(d_{1}+\nu\right)<a \nu$. Furthermore, $E_{1}$ is asymptotically stable under

$$
a \nu>\frac{b d_{2}\left(d_{1}+\nu+\alpha\right)^{2}}{d_{1}+\nu} .
$$

\section{APPENDIX C}

\section{Stability of Equilibria of (3.2)}

We now consider stability of (3.2). First, we find equilibria when $c=0$. When $c=0,(3.2)$ is reduced to

$$
\left\{\begin{array}{l}
\frac{d y_{1}}{d t}=\frac{a y_{2}(t)}{b+y_{2}(t)}-\left(d_{1}+\alpha\right) y_{1}(t)-\frac{a y_{2}(t-\tau)}{b+y_{2}(t-\tau)} e^{-\left(d_{1}+\alpha\right) \tau} \\
\frac{d y_{2}}{d t}=\frac{a y_{2}(t-\tau)}{b+y_{2}(t-\tau)} e^{-\left(d_{1}+\alpha\right) \tau}-d_{2} y_{2}(t)
\end{array}\right.
$$

It is easy to verify that $(\mathrm{A}-16)$ has a positive equilibrium $E_{10}=\left(y_{10}, y_{20}\right)$ with

$$
y_{20}=\frac{a e^{-\left(d_{1}+\alpha\right) \tau}}{d_{2}}-b, \quad y_{10}=\frac{a y_{20}}{\left(b+y_{20}\right)\left(d_{1}+\alpha\right)}\left(1-e^{-\left(d_{1}+\alpha\right) \tau}\right)
$$

if

$$
\frac{a e^{-\left(d_{1}+\alpha\right) \tau}}{d_{2}}>b .
$$

Note that the second equation can be separated from the first equation in (A16) and that the flow of the second equation of (A-16) is monotonic. It follows from Smith (1995) and Zhao (2003) that $E_{10}$ is globally stable whenever it exists. Furthermore, if the inequality in (A-17) is reversed, $(0,0)$ is globally stable for $(\mathrm{A}-16)$. 
For the reason of mathematical techniques, we consider the stability of $E_{1}=$ $\left(y_{10}, y_{20}, 0\right)$ for $(3.2)$, and leave the stability of $E_{0}=(0,0,0)$ to computer simulations. We linearize (3.2) at $E_{1}$ to obtain

$$
\begin{aligned}
\frac{d y_{1}}{d t}= & -\left(d_{1}+\alpha\right) y_{1}(t)+\frac{a b}{\left(b+y_{20}\right)^{2}} y_{2}(t)-\frac{a b e^{-\left(d_{1}+\alpha\right) \tau}}{\left(b+y_{20}\right)^{2}} y_{2}(t-\tau) \\
& +\left(\alpha y_{10}-\frac{a y_{20}^{2}}{\left(b+y_{20}\right)^{2}}\right) c(t)-\frac{\alpha a y_{20} e^{-\left(d_{1}+\alpha\right) \tau}}{b+y_{20}} \int_{t-\tau}^{t} c(\theta) d \theta \\
& +\frac{a y_{20}^{2} e^{-(d+\alpha) \tau}}{\left(b+y_{20}\right)^{2}} c(t-\tau), \\
\frac{d y_{2}}{d t}= & \frac{a b e^{-\left(d_{1}+\alpha\right) \tau}}{\left(b+y_{20}\right)^{2}} y_{2}(t-\tau)+\frac{\alpha a y_{20} e^{-\left(d_{1}+\alpha\right) \tau}}{b+y_{20}} \int_{t-\tau}^{t} c(\theta) d \theta-d_{2} y_{2}(t) \\
& -\frac{a y_{20}^{2} e^{-(d+\alpha) \tau}}{\left(b+y_{20}\right)^{2}} c(t-\tau), \\
\frac{d c}{d t}= & w c(t)\left(-\frac{a y_{20}}{\left(b+y_{20}\right)^{2}}+\alpha \frac{y_{10}}{y_{20}}\right) .
\end{aligned}
$$

If

$$
\frac{a y_{20}}{\left(b+y_{20}\right)^{2}}>\alpha \frac{y_{10}}{y_{20}}
$$

it follows from the third equation of (A-18) that $c(t) \rightarrow 0$ as $t \rightarrow \infty$. By the theory of asymptotically autonomous differential equations, we see that $E_{1}$ is asymptotically stable for (3.2). Furthermore, it is easy to see that $E_{1}$ is unstable if we have

$$
\frac{a y_{20}}{\left(b+y_{20}\right)^{2}}<\alpha \frac{y_{10}}{y_{20}}
$$

${ }_{411} \quad$ For the existence of positive equilibrium of (3.2), we have

Theorem B: (3.2) admits a unique positive equilibrium $E^{*}=\left(y_{1}^{*}, y_{2}^{*}, c^{*}\right)$ if and only if

$$
\left\{\begin{array}{l}
\frac{a}{b d_{2}}>e^{d_{1} \tau} \\
\frac{\alpha+d_{1}}{\alpha e^{-\left(\alpha+d_{1}\right) \tau}+d_{1}}>\frac{a e^{-\left(\alpha+d_{1}\right) \tau}}{b d_{2}} .
\end{array}\right.
$$

\section{Proof:}

Set $E=e^{-\left(d_{1}+\alpha s\right) \tau}$ with $s=1-c$. Then we have

$$
\begin{aligned}
& \alpha \frac{y_{1}}{y_{2}}=a \frac{y_{2}}{\left(b s+y_{2}\right)^{2}}, \\
& a \frac{s y_{2}}{b s+y_{2}}(1-E)=\left(d_{1}+\alpha s\right) y_{1}, \\
& a \frac{s y_{2}}{b s+y_{2}} E=d_{2} y_{2} .
\end{aligned}
$$


To get equations for $s$, we solve $y_{2}$ from (A-24) to obtain

$$
y_{2}=\frac{a E-b d_{2}}{d_{2}} s .
$$

It follows from (A-22) that

$$
y_{1}=\frac{a y_{2}^{2}}{\alpha\left(b s+y_{2}^{2}\right)^{2}}=\frac{\left(a E-b d_{2}\right)^{2}}{a \alpha E^{2}} .
$$

Substituting (A-25) and (A-26) into (A-23), we obtain

$$
a s(1-E)=\left(d_{1}+\alpha s\right) \frac{a E-b d_{2}}{\alpha E} .
$$

It follows that

$$
\frac{a E}{b d_{2}}=\frac{\alpha s+d_{1}}{\alpha s E+d_{1}} .
$$

To derive conditions such that (A-27) admits a unique solution in $(0,1)$, we define

$$
f(s):=\frac{a E}{b d_{2}}, \quad g(s):=\frac{\alpha s+d_{1}}{\alpha s E+d_{1}} .
$$

Note that $f(s)$ is a decreasing function of $s$ and has a maximum $f(0)=\frac{a}{b d_{2}} e^{-d_{1} \tau}$. Since $E^{\prime}=-\alpha \tau E$, we have

$$
\begin{aligned}
g^{\prime}(s) & =\frac{1}{\left(\alpha s E+d_{1}\right)^{2}}\left\{\alpha\left(\alpha s E+d_{1}\right)-\left(\alpha s+d_{1}\right)(\alpha E-\alpha s \cdot \alpha \tau E)\right\} \\
& =\frac{1}{\left(\alpha s E+d_{1}\right)^{2}}\left\{\alpha d_{1}(1-E)+\left(\alpha s+d_{1}\right) \alpha s \cdot \alpha \tau E\right\}>0 .
\end{aligned}
$$

Hence, (A-27) admits a unique solution $s^{*}$ in $(0,1)$ if and only if $f(0)>g(0)$ and $f(1)<g(1)$. The former corresponds to the first inequality of (A-21), while the latter corresponds to the second inequality of (A-21). From (A-27), we see that

$$
a E\left(s^{*}\right)=\frac{\alpha s^{*}+d_{1}}{\alpha s^{*} E\left(s^{*}\right)+d_{1}} b d_{2}>b d_{2} .
$$

It follows from $(\mathrm{A}-25)$ and $(\mathrm{A}-26)$ that when $c=c^{*}=1-s^{*}$, we have

$$
y_{2}^{*}=\frac{a E\left(s^{*}\right)-b d_{2}}{d_{2}} s^{*}>0, \quad y_{1}^{*}=\frac{\left(a E\left(s^{*}\right)-b d_{2}\right)^{2}}{a \alpha E^{2}\left(s^{*}\right)}>0 .
$$

This completes the proof.

Note that the first inequality of (A-21) is satisfied if (A-17) holds. Further, it is easy to see that the second condition in (A-21) is equivalent to (A-20). Further, (A-21) can be rewritten as

$$
\tau^{*}:=\frac{1}{d_{1}+\alpha} \ln \frac{a d_{1}+\sqrt{\left(a d_{1}\right)^{2}+4 a \alpha b d_{2}\left(\alpha+d_{1}\right)}}{2 b d_{2}\left(\alpha+d_{1}\right)}<\tau<\frac{1}{d_{1}} \ln \frac{a}{b d_{2}}:=\tau^{* *} .
$$


The left inequality of $(\mathrm{A}-28)$ ensures that $E_{1}=\left(y_{10}, y_{20}, 0\right)$ is unstable. If we define

$$
\tau_{0}=\frac{1}{d_{1}+\alpha} \ln \frac{a}{b d_{2}},
$$

by summarizing above discussions, we obtain Table 2 that presents the distribution of equilibria of (3.2).

\section{References}

P. A. Abrams. The evolution of predator?cprey interactions: theory and evidence. Annu. Rev. Ecol. Syst., 31:79-105, 2000.

P. A. Abrams. Habitat choice in predator-prey systems: spatial instability due to interacting adaptive movements. American Naturalist, 169:581-594, 2007.

P. A. Abrams, R. Cressman, and V. Krivan. The role of behavioral dynamics in determining the patch distributions of interacting species. American Naturalist, 169:505-518, 2007.

W. Aiello and H. Freedman. A time delay model of single- species growth with stage structure. Mathematical Biosciences, 101:139-153, 1990.

W. Aiello, H. Freedman, and J. Wu. Analysis of a model representing stagestructured population growth with state-dependent time delay. SIAM J. Appl. Math., 52:855-869, 1992.

A. B. Bruno, G. S. Requena, E. G. Martins, and G. Machado. Effects of maternal care on the lifetime reproductive success of females in a neotropical harvestman. Journal of animal ecology, 76:937-945, 2007.

A. M. de Roos, L. Persson, and H. R. Thieme. Emergent allee effects in top predators feeding on structured prey populations. Proc. R. Soc. Lond. B., 270: 611-618, 2003.

D. L. DeAngelis, M. Vos, W. M. Mooij, and P. A. Abrams. Feedback effects between the food chain and induced defense strategies. Fundamental ecology: from energetics to food webs. Springer, Netherlands, 2006.

U. Dieckmann and R. Law. The dynamical theory of coevolution: a derivation from stochastic ecological processes. J. Math. Biol., 34:579-612, 1996. 
L. S. Forbes and D. W. Mock. Parental optimism and progeny choice: When is screening for offspring quality affordable? J. Theor. Biol., 192:3-14, 1998.

S. A. H. Geritz, E. Kisdi, G. Meszéna, and J. A. J. Metz. Adaptive Dynamics of Speciation: EcologicalUnderpinnings. In: Adaptive Speciation, eds. by U. Dieckmann, M. Doebeli, J. A. J. Metz and D. Tautz, pp. 54-75. Cambridge University Press, 2004.

S. A. Gourley and Y. Kuang. A stage structured predator-prey model and its dependence on maturation delay and death rate. J. Math. Biol., 49:188-200, 2004.

S. A. Gourley and Y. Kuang. A delay reaction-diffusion model of the spread of bacteriophage infection. SIAM J. Appl. Math., 65:550-566, 2005.

J. Hofbauer and K. Sigmund. Evolutionary Games and Population Dynamics. Cambridge University Press, 1998.

A. I. Houston, T. Székely, and J. M. McNamara. Conflict between parents over care. Trends in Ecology and Evolution, 20:33-38, 2005.

T. Kostova, J. Li, and M. Friedman. Two models for competition between age classes. Math. Biosci., 157:65-89, 1999.

V. Krivan. The Lotka-Volterra predator-prey model with foraging-predation risktrade-offs. American Naturalist, 170:771-782, 2007.

C. M. Lessells. A theoretical framework for sex-biased parental care. Animal Behaviour, 56:395-407, 1998.

J. A. J. Metz, R. M. Nisbet, and S. A. H. Geritz. How should we define "fitness" for general ecological scenarios? Trends in Ecology and Evolution, 7:198-202, 1992.

R. M. Nisbet and W. S. C. Gurney. The systematic formulation of population models for insects with dynamically varying instar duration. Theor. Pop. Biol., 23:114-135, 1983.

J. D. Reynolds, N. B. Goodwin, and R. P. Freckleton. Evolutionary transitions in parental care and live bearing in vertebrates. Philosophical Transactions of the Royal Society B: Biological Sciences, 357:269-281, 2002. 
H. Seno and H. Endo. A mathematical model on the optimal timing of offspring desertion. J. Theor. Biol., 246:555-563, 2007.

H. L. Smith. Monotone Dynamical Systems. An introduction to the theory of competitive and cooperative systems. Math Surveys and Monographs 41. American Mathematical Society, Providence, RI, 1995.

Y. Takeuchi, W. Wang, and Y. Saito. Global stability of population models with patch structure. Nonlinear Anal. Real World Appl., 7:235-247, 2006.

H. R. Thieme. Mathematics in Population Biology. Princeton University Press, Princeton, NJ, 2003.

W. Wang and L. Chen. A predator-prey system with stage-structure for predator. Comput. Math. Appl., 33:83-91, 1997.

W. Wang, G. Mulone, F. Salemi, and V. Salone. Permanence and stability of a stage-structured predator-prey model. J. Math. Anal. Appl., 262:499-528, 2001.

W. Wang, S. Nakaoka, and Y. Takeuchi. Invest conflicts of adult predators. $J$. Theor. Biol., (to appear), 2008.

W. Wang, Y. Takeuchi, Y. Saito, and S. Nakaoka. Prey-predator system with parental care for predators. J. Theor. Biol., 241:451-458, 2006.

W. Wang and X. Zhao. An age-structured epidemic model in a patchy environment. SIAM J. Appl. Math., 65:1597-1614, 2005.

W. Wang and X. Zhao. an epidemic model with population dispersal and infection period. SIAM J. Appl. Math., 66:1454-1472, 2006.

O. Yaniv. An asymmetric parental investment conflict with continuous strategy sets. J. Theor. Biol., 237:245-256, 2005.

X. Zhao. Dynamical systems in population biology. CMS Books in Mathematics, 16. Springer-Verlag, New York, 2003. 